$$
\text { قلبة الجب البيطرى والو لادة جامعة البعث ـ سوريا }
$$

\title{
OMENTOPEXY BY UTRECHT'S METHOD FOR TREATMENT OF LEFT DISPLACEMENT OF THE ABOMASUM IN COWS
}

(With 3 Tables and 4 Figures)

$$
\text { By }
$$

\section{S. HADAD and A. AOMARI}

(Received at 21/11/2011)

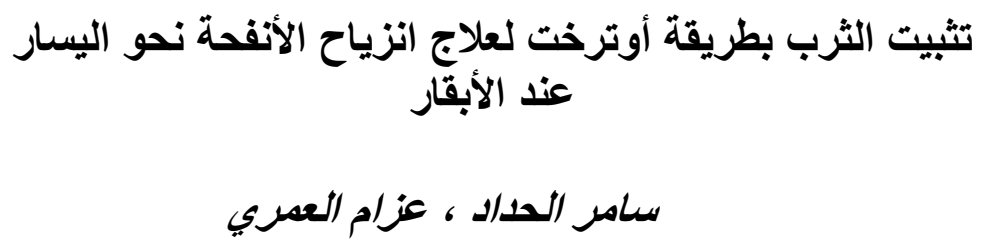

\section{SUMMARY}

The study was carried out on fifteen cows with left displacement of abomasum, which diagnosed between one to six weeks postparturient by simultaneous percussion and auscultation of the paralumbar left area and last left ribs where "ping" sound appear. Left displacement of abomasum was associated with other intercurrent diseases like: metritis, mastitis, lameness, bronchitis and diarrhea. Left displacement of abomasum was corrected surgically using Utrecht's method under the effect of low paraventral analgesia in standing position. Some clinical and biochemical examination had performed before and after surgical treatment. The results revealed that this method is an effective technique, safe and fast to use in the field without complications, in addition cows returned to its normal state in short time after surgery.

Key words: Utrecht's method, displacement of the abomasums, cows. 


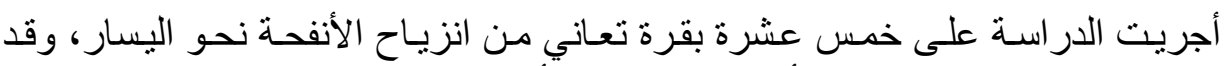

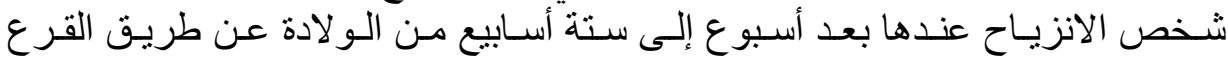

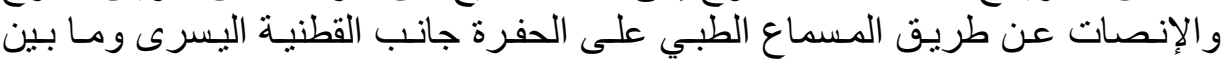

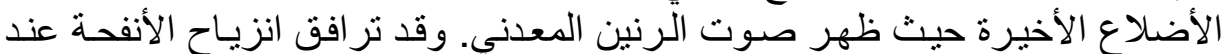

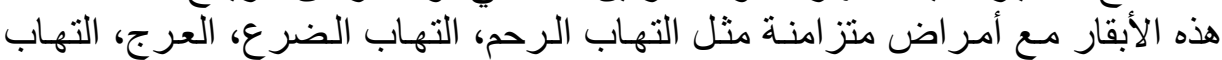

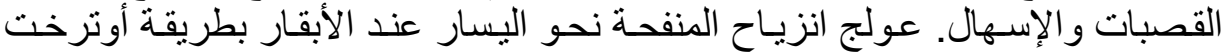

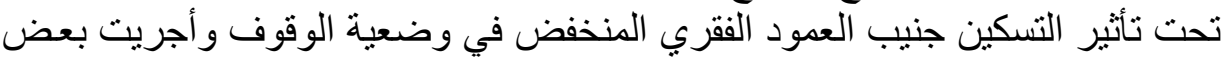

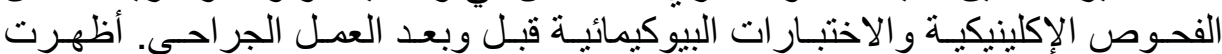

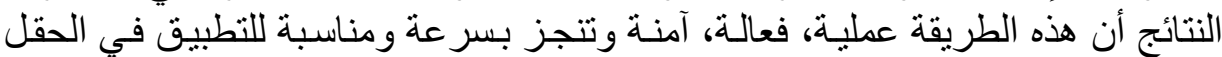
دون حدوث مضاعفات وعادت الأبقار بعد العمل الجر احي إلى وضعها الطبيعي في

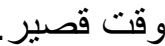

الكلمات المفتاحية: انزياح الأنفحة ، أوترخت

\section{INTRODUCTION}

مقدمــة

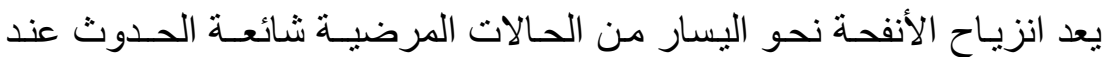

الأبقار الحلوب عاليـة الإنتاج Guzelbektes et،Naeini and Rowshan, 2008

(Staric et al., 2010 ، al., 2010

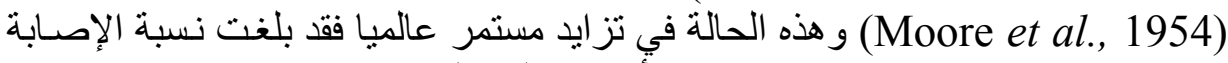

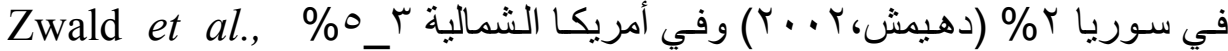

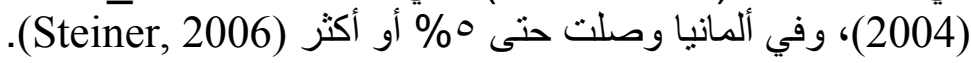

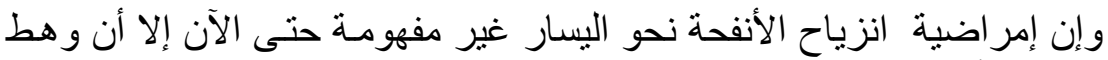

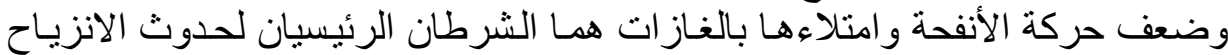

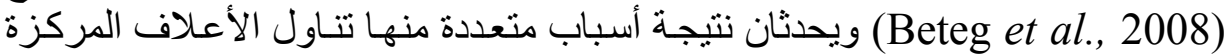

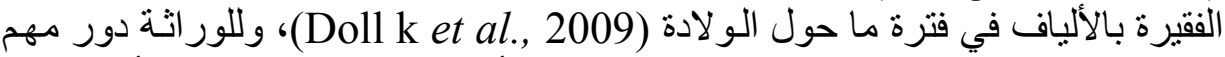

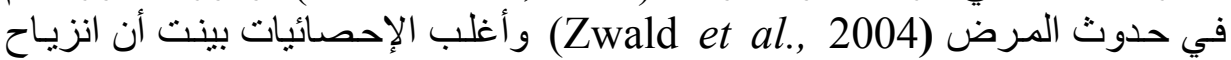

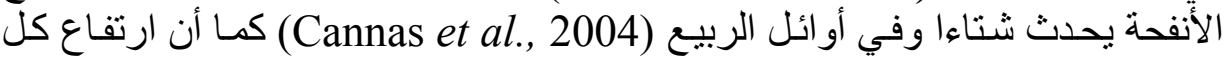

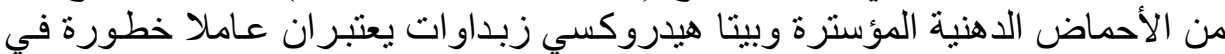

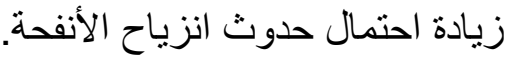

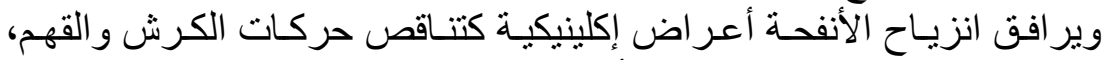

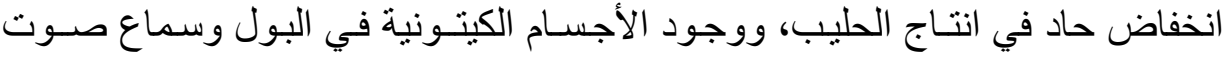

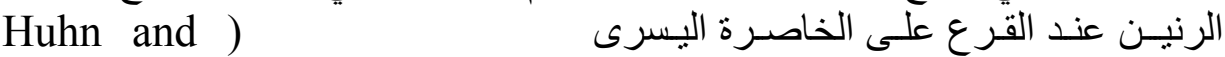
كما وير افق انزياح الأنفحة تغيرات في (Radostits et al., 2000 ، Nelson,1995

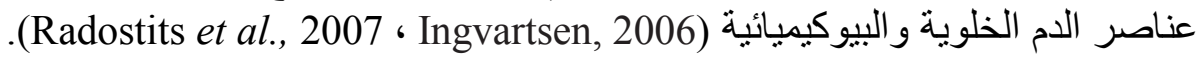




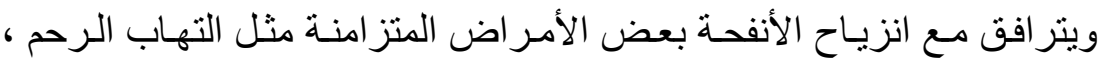

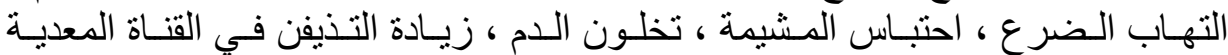

المعوية (Burim et al., 2010 ، Steiner, 2006)

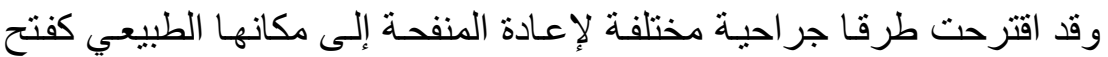

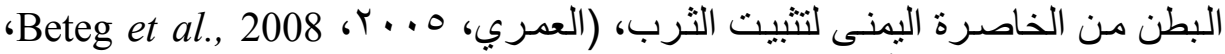

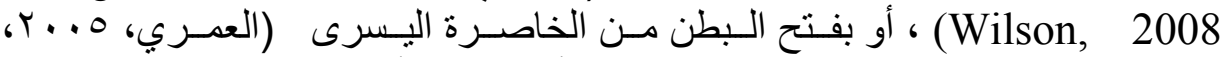

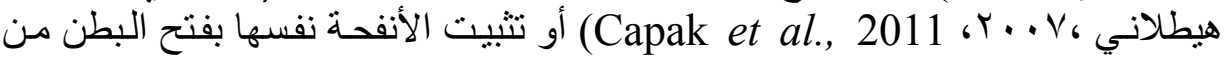
الخط الوسطي الأيمن (Baird and Harrison, 2001) أو باستخدام تقنيـة منظـار البطن في وضعية الوقوف أو الاستلقاء (Christiansen, 2004).

أهدف البحث Objectives of the Study:

فتح البطن من الخاصرة اليسرى لتثبيت الثرب بطريقة أوترخت عند الأبقار المصابة بانزياح الأنفحة نحو اليسار.

\section{MATERIALS and METHODS مواد وطرائق العمل}

أجري البحث في المشفى التعليمي بكلية الطب البيطري-جامعة البعث سورية

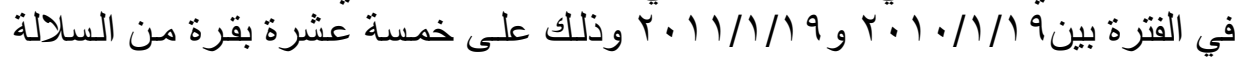

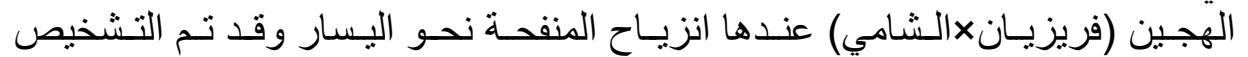

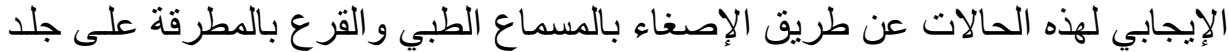

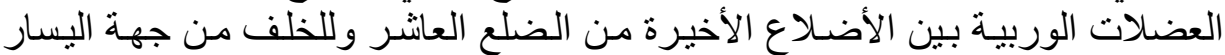

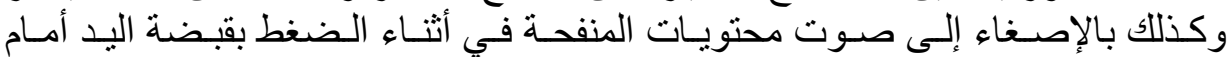

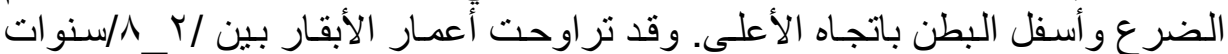

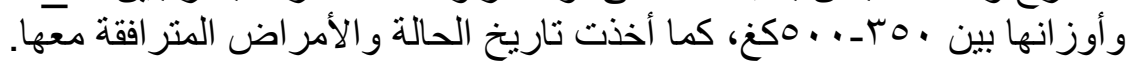

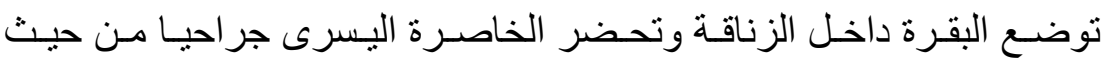

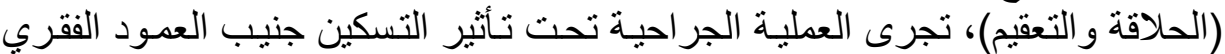

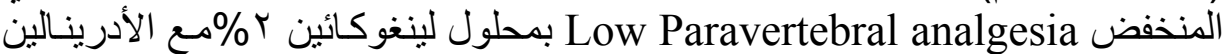
(Norbrook) Lignocaine and Adrenaline الجر احي. يجرى الثق الجراحي بشكل عمودي على بعد حو الي ه سم أسفل الفقرات

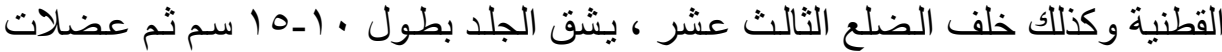

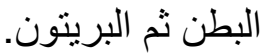

تمد اليد لاستقصاء التجويف البطني حيث توجد المنفحة بين الكرش وجدار

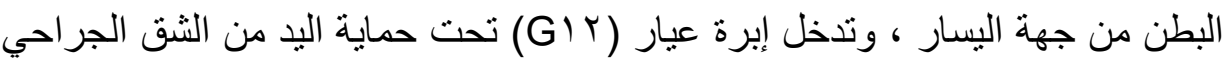


ثم تدخل في الانحناء الكبير للمنفحة وتفرغ من الغاز ات ثم تسحب الإبرة وبعدها يسحب

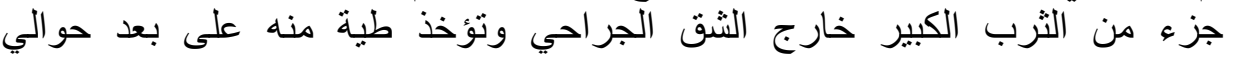

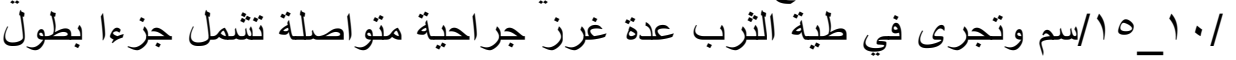

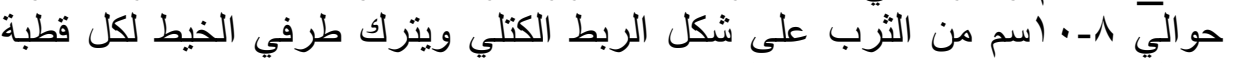

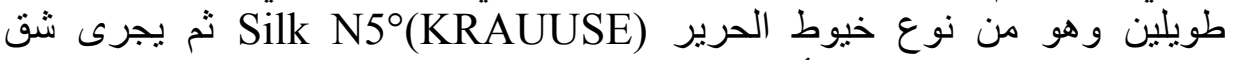

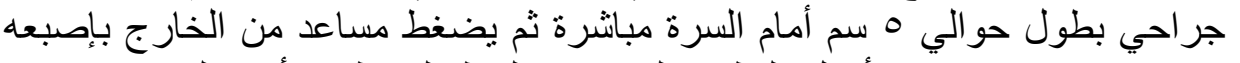

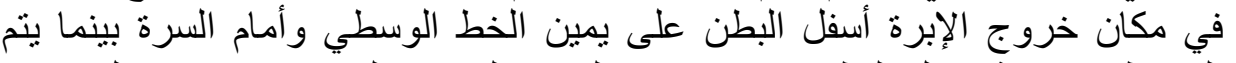

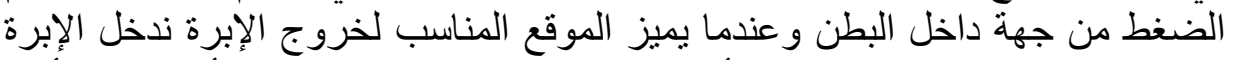

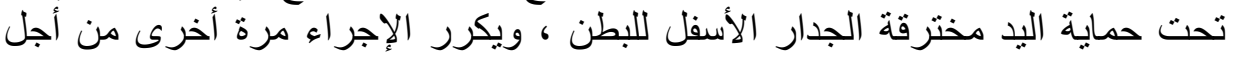

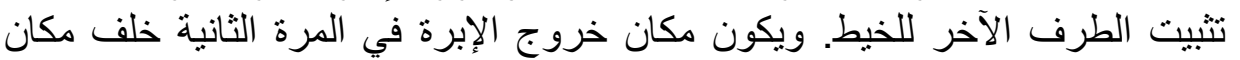

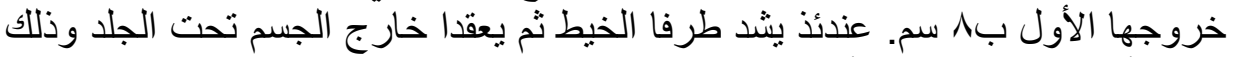

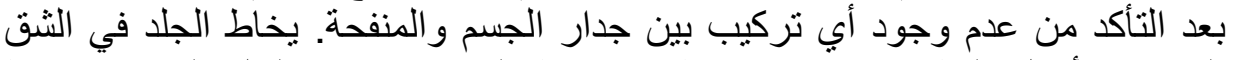

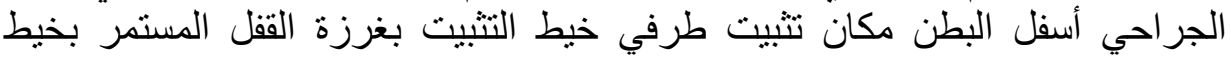
الحرير رقم Silk No 3\4(KRAUUSE ثم يوضع على الجرح بخاخ صاد حيوي ئا أما الثق الجراحي على الخاصرة اليسرى فيخاط البريتون

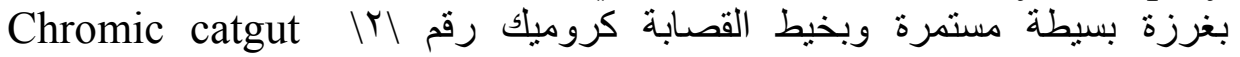
USP2,Metric6 ثم تخاط العضلات البطنية بالخيط السابق الذكر وبالقطب المتصالبة ، ثم يخاط الجلد بغرزة القلل المستمر بخيط الحرير رقم Silk No (KRAUUSE) 3\4 ثم يوضع على الجرح بخاخ صاد حيوي Allamycine spray.

Betamox تعطى البقرة حقنا بالعضل الصادين الحيويين أموكسوسيللين

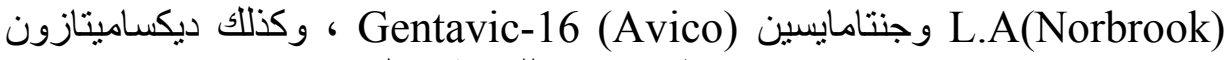

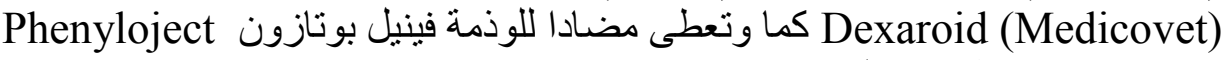

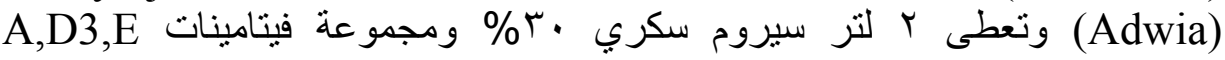
بالإضافة إلى فيتامين C، ويعالج الحماض أو القلاء بالإضافة إلى تخلون الدام.

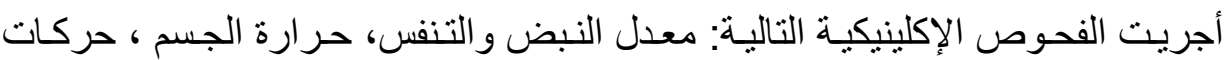

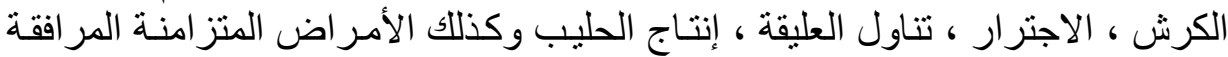

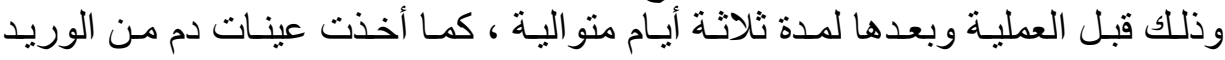
الوداجي لحساب العدد الكلي للكريات الحمر و البيض بطريقة التهات عداد (نيوبار ).

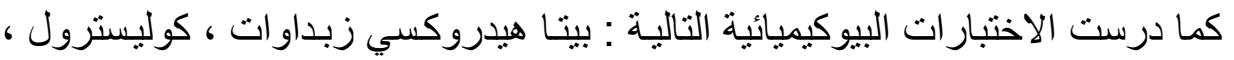

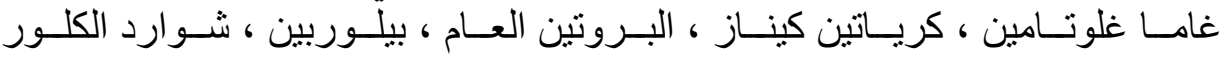

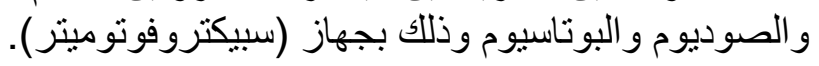

\section{RESULTS}




\section{التتــائــجـ}

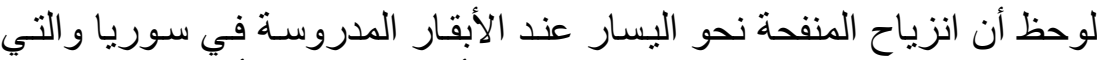

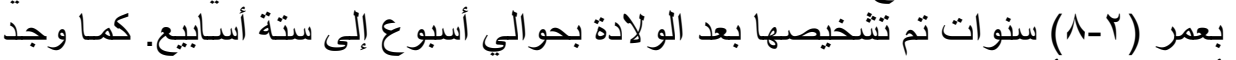

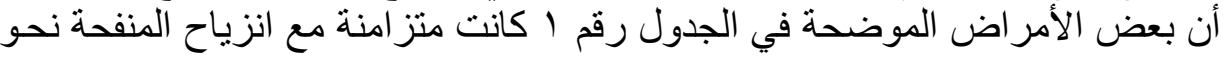

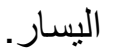

جدول رقم ا : يبين الأمر اض المنزامنة مع انزياح المنفحة نحو اليسار عند الأبقار

\begin{tabular}{|c|c|}
\hline العدد العد & الأمر اض المتر اققة \\
\hline 0 & التهاب رحم \\
\hline$\varepsilon$ & التهاب ضرع \\
\hline$r$ & إسهال \\
\hline 1 & عرج \\
\hline$r$ & التهاب قصبات \\
\hline 10 & المجموع \\
\hline
\end{tabular}

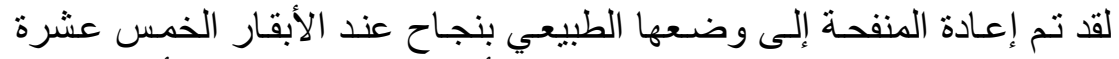

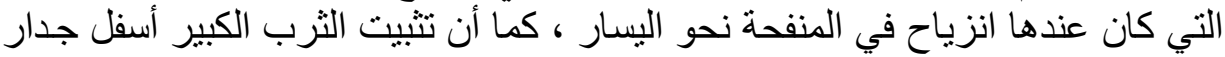

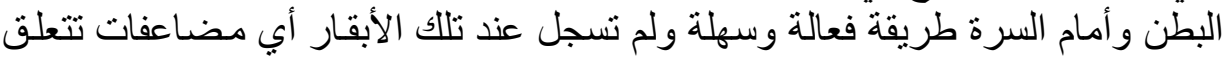

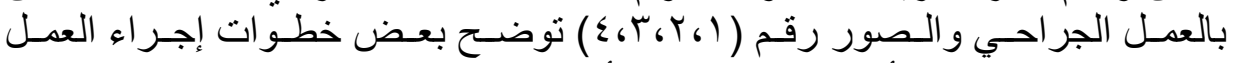
الجر احي لتنبيت الثثرب أسفل البطن بطريقة أوترخت 

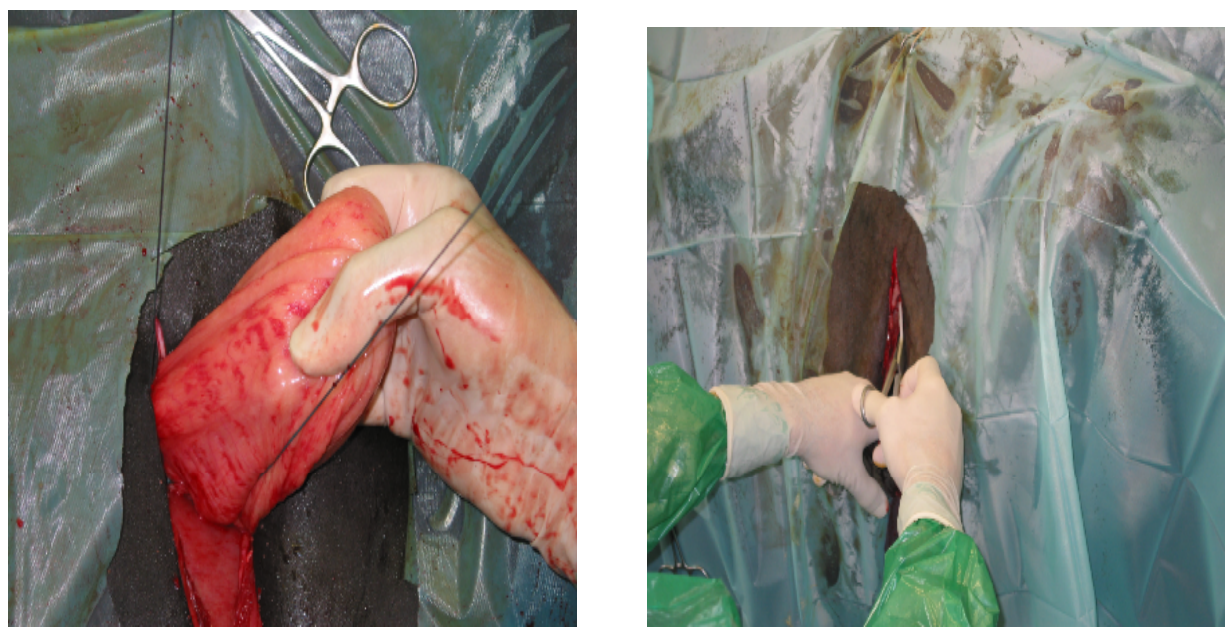

الصورة رقم ب : توضح سحب الثرب الكبير من الثق الجر احي وخياطته بخيوط توضئ الحرير

$$
\text { الصورة رقم ال: الخى في الجرة اليسرى الثقى }
$$

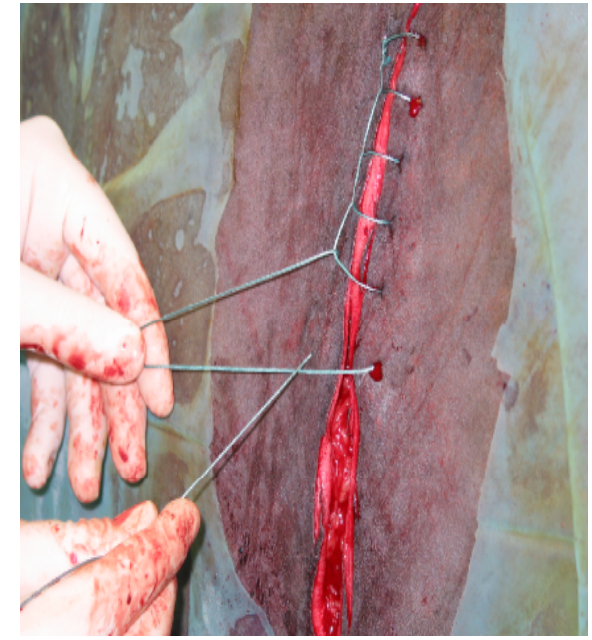

صورة رقم ع : توضح إغلاق الثق الجر احى بطريقة القفل المستمر

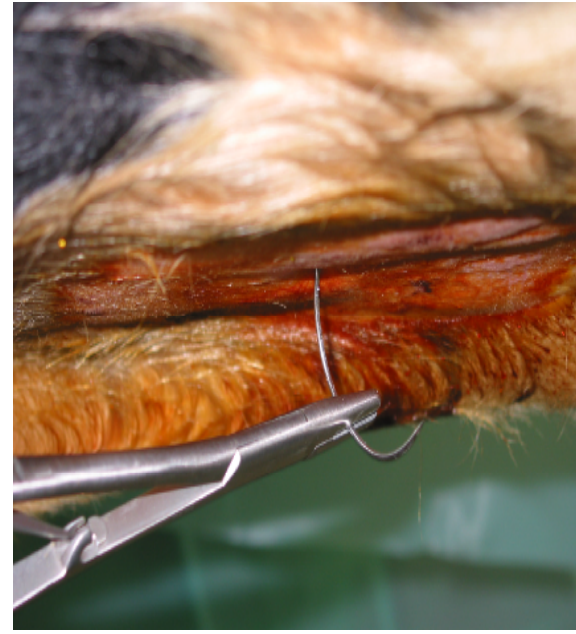

صورة رقم ب: توضح مكان خروج الإبرة أبرة

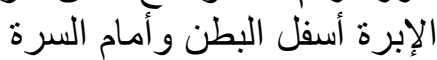

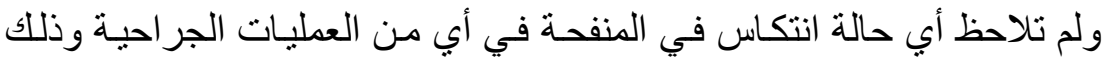

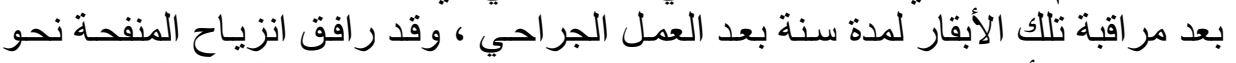

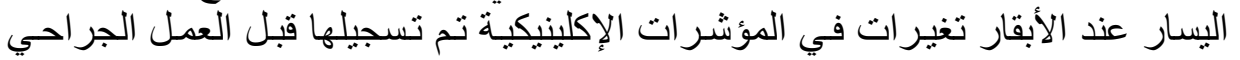

وبعده كما في الجدول رقم (ب) الإن 
جدول رقم ץ: يبين التغير ات الإكلينيكية المر افقة لانزياح المنفحة نحو اليسار قبل وبعد

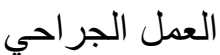

\begin{tabular}{|c|c|c|c|c|}
\hline \multicolumn{4}{|c|}{ 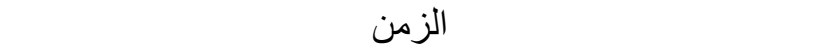 } & \multirow[b]{2}{*}{ المؤشرات } \\
\hline آل أيام بعد & آبو مبلية & العملية & قبل العملية & \\
\hline $7,0 \pm 7 V, 0$ & $1 r, 0 \pm T V, r$ & $1 \cdot, q \pm \vee \vee, r$ & $v, r \pm v 1, l$ & معدل النبض \\
\hline$\neg, r \pm r \wedge, \vee$ & \urcorner$, r \pm r \cdot, q$ & $0, \Sigma \pm Y \wedge, T$ & $0, \wedge \pm Y V, \wedge$ & معدل التنفس \\
\hline$\cdot, \leq \pm \Gamma \wedge, \tau$ & $\cdot, 0 \pm \mu \wedge, 7$ & $\cdot, 7 \pm r \wedge, q$ & $\cdot, v \pm r q, r$ & حر ارة الجسم م \\
\hline 1,$\urcorner \pm r, \wedge$ & $1, V \pm r, r$ & $1,0 \pm 1, V$ & $1, \varepsilon \pm 1, \Gamma$ & دقائق \\
\hline$\cdot, 0 \pm r, q$ & $\cdot, 0 \pm r, q$ & $\cdot, \varepsilon \pm r, 0$ & $1, r \pm 1, \varepsilon$ & كمية الروث \\
\hline $1, \cdot \pm r, V$ & $1, r \pm r, \varepsilon$ & $1,1 \pm 1, V$ & $1, \Sigma \pm 1, r$ & كمية العليقة \\
\hline$\varepsilon, 9 \pm 11,1$ & $r, \cdot \pm r, r$ & $r, \wedge \pm\urcorner, 1$ & $\varepsilon, r \pm \vee, \varepsilon$ & إنتاج الحليب كغاباليوم \\
\hline
\end{tabular}

ملاحظة: في الجدول رقم (r)

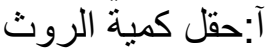

الرقم ا :يعني خروج كمية الروت الروث بكمية قليلة

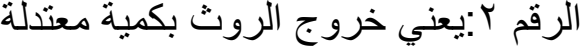

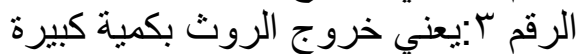
ب:مقل كمية العليقة الرقم ا:يعني تناول العليقة بكمية قليلة

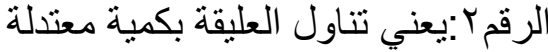
الرقمب:يعني تناول العليقة بكمية جيدة

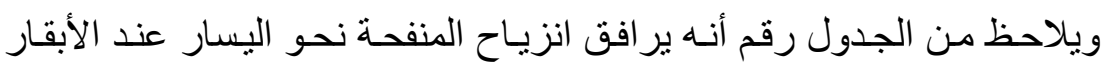

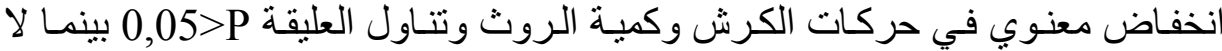

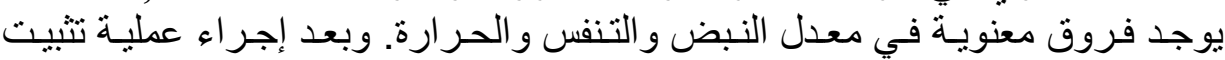

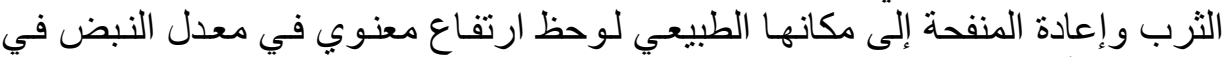

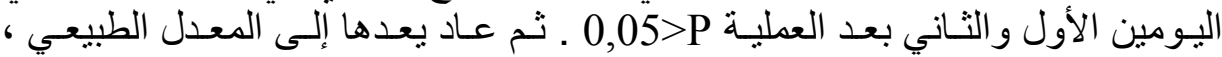

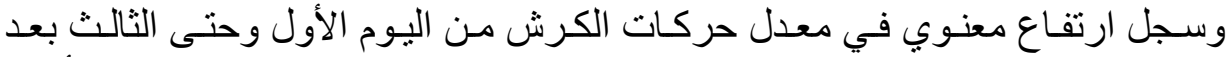

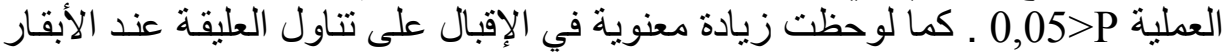
بعد العملية 0,05>P ، وكان هناك انخفاض غير معنوي في انتاج الحليب بعد العمل

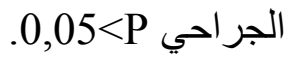


وسجلت تغير ات في بعض معدلات مكونـات الدم البيوكيميائيسة رافقت انزيـاح الجياح

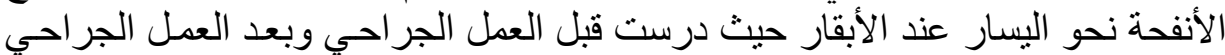

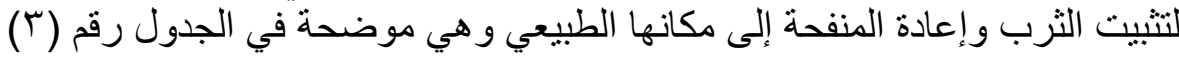

جدول رقمّا: يبين التغيرات في بعض معدلات الدم البيوكيميائية عند الأبقار التي عندها

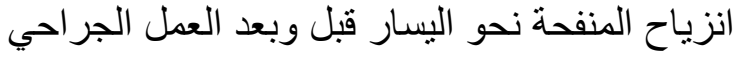

\begin{tabular}{|c|c|c|c|c|}
\hline \multicolumn{4}{|c|}{ الزمن } & \multirow[b]{2}{*}{ المعايير } \\
\hline r أيام بعد & آليوم بعد & يوم بعد العملية & قبل العملية & \\
\hline $9 . \vee \pm 71.0$ & $1 \cdot . r \pm 70.9$ & $9.9 \pm 7 V .7$ & $\left.1 Y_{.} \leqslant \pm V V_{.}\right)$ & غالبـروتين العـام \\
\hline $1 . r \pm r . \Sigma$ & 1.r土T. & $1 . r \pm r .0$ & $1.9 \pm \varepsilon .7$ & مولالـــــة ميلـــي \\
\hline $1 \Lambda . r \pm Y \circ \Lambda$ & 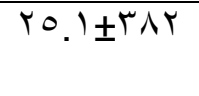 & $\varepsilon r . \wedge \pm \neg T r$ & $r r_{.} \varepsilon \pm r_{0} 01$ & وحريـاتين كينـاز \\
\hline $11 . \wedge \pm 1 \leqslant . \Gamma$ & $1 Y .0 \pm 10.1$ & $1 r .9 \pm 1 V .1$ & 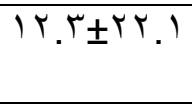 & ميكرومول/J بيلـين \\
\hline$r . r \pm 0 \leqslant . r$ & $\varepsilon . \pm 0 \leqslant . \Gamma$ & $V . I \pm 0 V . r$ & $0.1 \pm 09.1$ & وحدة دولية/لــالمين \\
\hline$\because \Psi \pm \cdot \varepsilon$ & $\cdot r \pm \cdot r$ & $\because \Psi \pm . \Sigma$ & $r . \wedge \pm r . r$ & 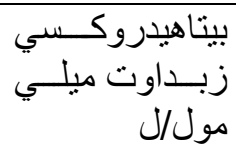 \\
\hline$\cdot \wedge \pm Y$. & $1 . \pm \pm .1$ & $1.1 \pm 1.9$ & $1.1 \pm Y . Y$ & كوليـي مول/لترول \\
\hline$\varepsilon . \wedge \pm 1 \uparrow \wedge . q$ & $\varepsilon . \wedge \pm 1 \leqslant 1.1$ & $r . V \pm 1 r q .7$ & $\Gamma .0 \pm \mid r \wedge .1$ & مول /لصوديوم ميلي \\
\hline$\because V \pm r . \varepsilon$ & $.7 \pm r . \varepsilon$ & $\cdot 0 \pm r . \varepsilon$ & $\cdot{ }^{\top} \pm r . r$ & البوتاسيوم ميلِي \\
\hline$\varepsilon .1 \pm 1 \cdot 7 . Y$ & $r .0 \pm 1 \cdot v .1$ & $\{.1 \pm 1 \cdot 7.0$ & $0.7 \pm 1 \cdot 0 . r$ & مول/لــور ميالـــي \\
\hline$\because V \pm 0.7$ & $.9 \pm 0.7$ & $\because V \pm 0 . V$ & $\because \wedge \pm 0 . \wedge$ & 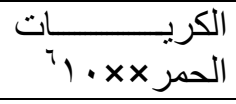 \\
\hline$r .0 \pm 7.9$ & $r . \wedge \pm V .1$ & 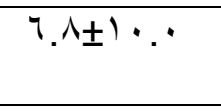 & $r .0 \pm V .0$ & 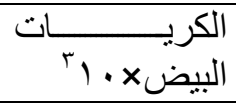 \\
\hline
\end{tabular}

من الجدول رقم (ب ) يلاحظ أن انزياح المنفحة نحو اليسار عند أبقار الدراسـة

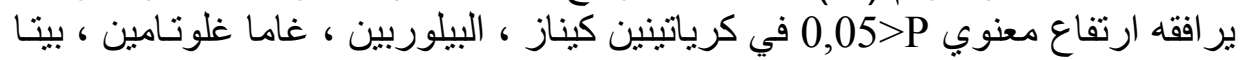




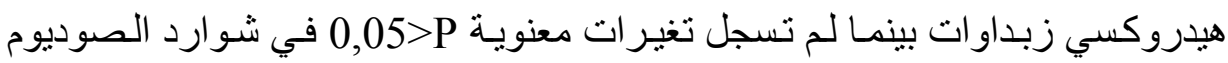
و البوتاسيوم و الكلور و البروتين العام و اليوريـا ، و الكوليسترول ،وتيرات العدد الكلي للكريـات

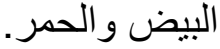

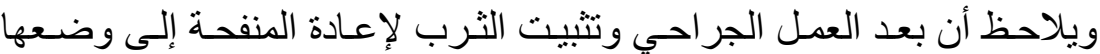

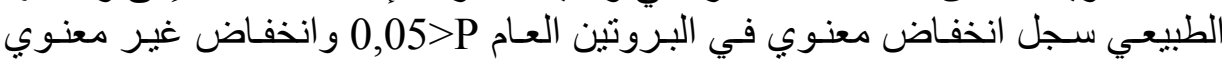

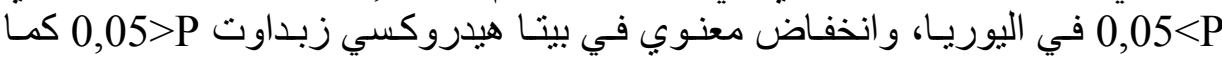

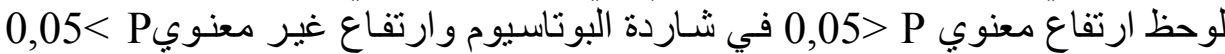

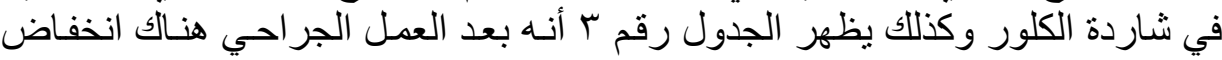
غير معنوي ن >0,05 في العدد الكلي للكريات الحمر.

\section{DISCUSSION}

المناقشـة

إن انزياح المنفحة نحو اليسار يلاحظ عند الأبقار بشكل شائع في فترة مـا حول

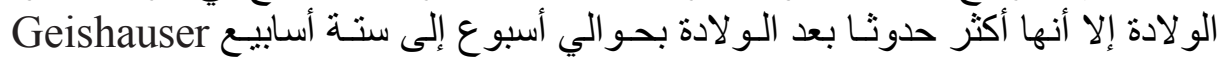
Wittek et al., ‘Zadnik, 2003 ، Eicher et al., 1999 ،et al., 1995) (Staric et al., 2010 ، وهذه النتائج تتفق مـع تاريخ الحالة التي دونت عند 2005 الأبقار التي أجريت عليها الدراسة.

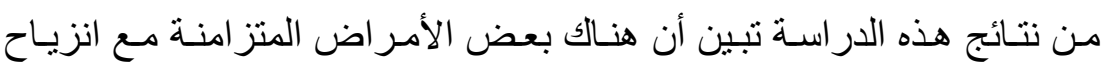

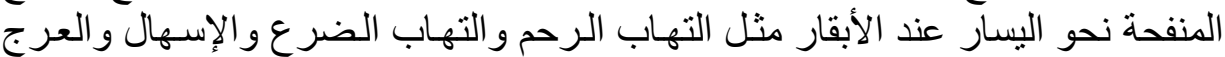

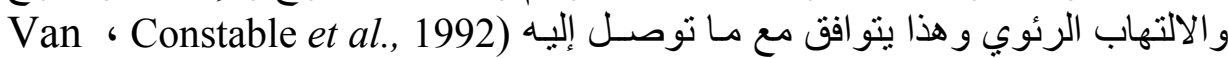
.(Beteg et al., 2008 ، Winden and Kuiper, 2003

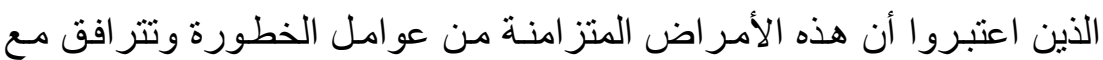

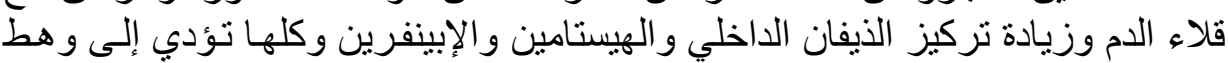

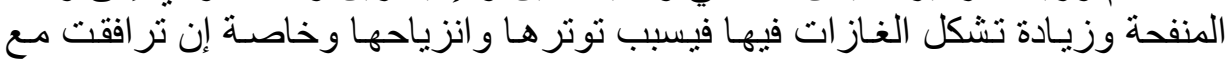

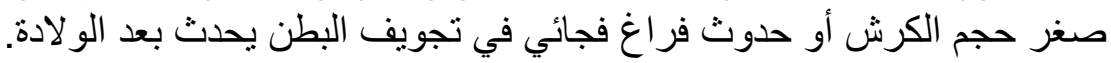

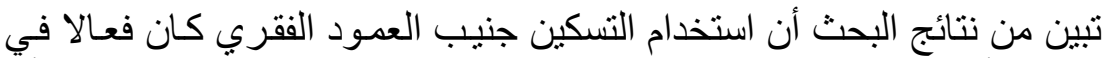

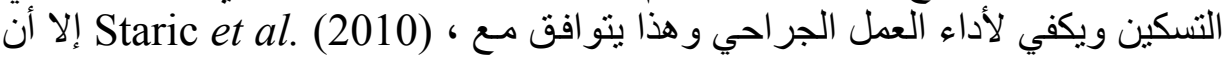
قد استعمل الكسيلازين مـع البوترفانول بالإضـافة إلى التسكين

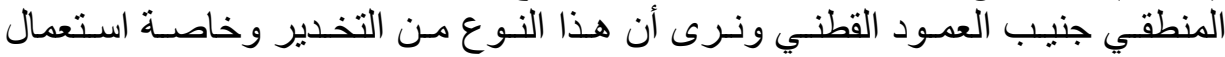

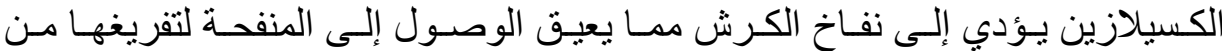

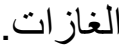

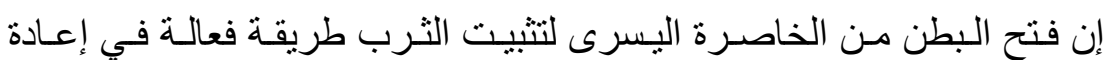

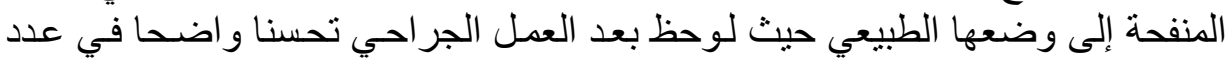

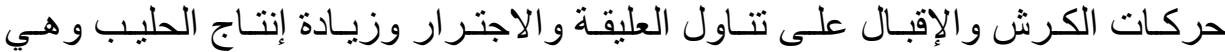

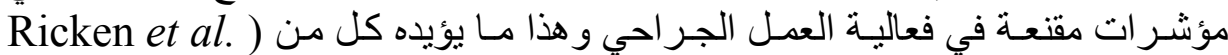




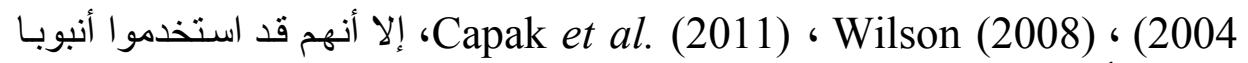

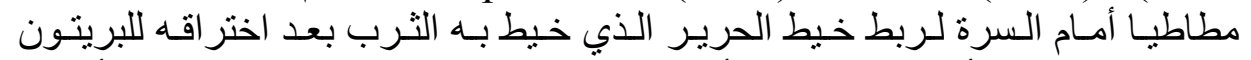

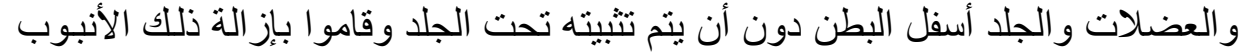

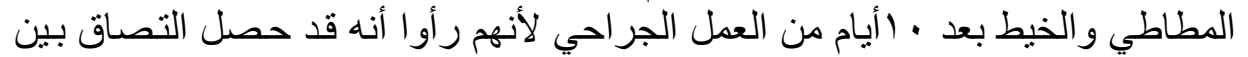
الثرب الكبير و البريتون في مكان التنبيت.

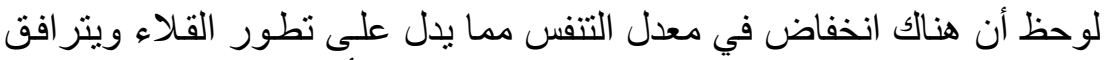

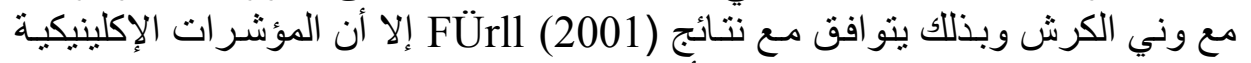
(الحرارة ، النبض ، التنفس) لم تعطي أي تغير ات ات معنوية.

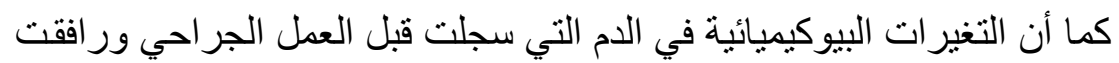

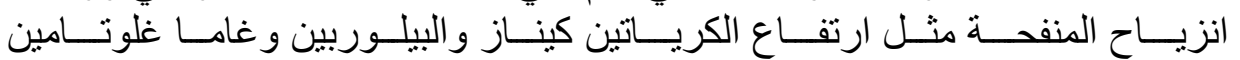

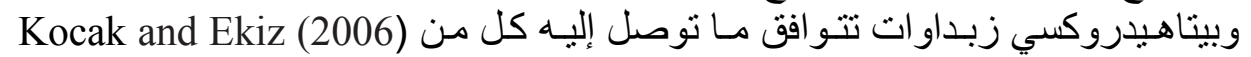
Van Winden and ) ، Saco and Gimenezm (2008)، Ingvartsen (2006)،

.Kuiper (2003

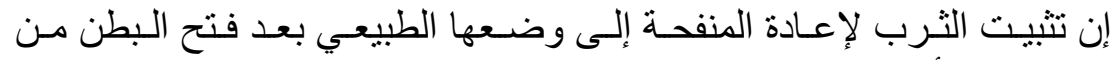

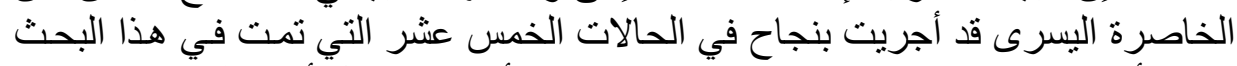

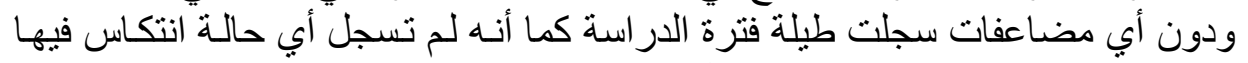

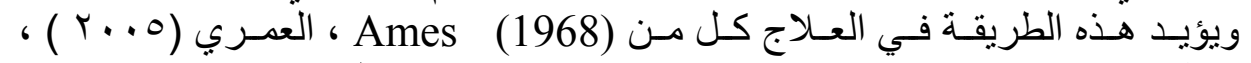

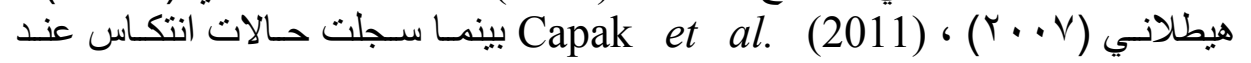
.Naeini and Rowshan (2008)

\section{REFERENCES}

Ames, S. (1968): Repositioning displaced abomasum in the cow. Javma, 153. 1470-1471.

Baird, A.N. and Harrison, S. (2001): Surgical treatment of left displaced abomasums. Compend Contin Educ Pract Vet. 23: 102-108.

Beteg, F.; Muste, A.; Oan, L.; Mates, N. and Ober, C. (2008): Clinical aspects and surgical treatmeant in left displacement of abomasums in Holstein cows, Lucrari Stllnlfice Medicin. Vol. XLI, 132-345.

Burim, N.; Ametaj, Qendrim Zebeli and Summera Iqbal. (2010): Nutrition, microbiota, and endotoxin-related diseases in dairy cows. R. Bras. Zootec., 39: 433-444.

Cannas da Silva, J.; Schauberger, G.; Rosario Oliveira, M.; Segao, S.; Kümper, H. and Baumgartner, W. (2004): Does the weather influence the occurrence of abomasal displacement in dairy cows? Deutsche Tiera"rztliche Wochenschrift 111: 51-57 
Capak, D. (2011): Modification to utrecht method of surgical-6 treatment of abomasal displacement Veterinarska Stanica 42 Supplement 2: 311-315.

Christiansen, K. (2004): Laparoskopisch kontrollierte Operation des nach links verlagerten Labmagens (Janowitz-Operation) ohne Ablegen des Patienten. Tierärz Prax, 32(5): 118-121.

Constable, P.D.; Miller, G.Y.; Hoffsis, G.F.; Hull, B.L. and Rings, D.M. (1992): Risk factors for abomasal volvulus and left abomasal displacement in cattle. American Journal of Veterinary Research 53: 1184-1192.

Wilson, D.G. (2008): Management of Abomasal Displacement. Volume 8, Issue 8. Large Animal Veterinary rounds.

Doll, K.; Sickinger, M. and Seeger, T. (2009): New aspects in the pathogenesis of abomasal displacement. The Veterinary Journal 181: 90-96.

Eicher, R.; Audige, L.; Braun, U.; Blum, J.; Meylan, M. and Steiner, A. (1999): Epidemiologie und Risikofaktoren der Blinddarmdilatation und Labmagenverlagerung bei der Milchkuh. Schweizer Archiv für Tierheilkunde 141: 423-429.

FÜrll, M. (2001): Dislocatio alomasi (DA) bei erwachsenen Rindern Sonderdruck aus Handlexikon der tieraztichen Praxis Enke Verlag Stuttgart.

Geishauser, T. (1995): Abomasal displacement in the bovine - a review on character, occurrence aetiology and pathogenesis, J. Vet. Med. Ser A, 45: 499-511.

Guzelbektes, H.; Sen, M. Ok; Constable, P.D.; Boydak, M. and Coskun, A. (2010): Serum Amyloid A and Haptoglobin Concentrations and Liver Fat Percentage in Lactating Dairy Cows with Abomasal Displacement, J. Vet. Intern. Med. 24: 213-219.

Huhn, J. and Nelson, D. (1995): Right sided abomasal problems in dairy cattle. Vet. Med. December.

Ingvartsen, K.L. (2006): Feeding and management-related diseases in the transition cow: p_hysiological adaptations around calving and strategies to reduce feeding-related diseases. Animal Feed Science and Technology, 126, 3-4: 175-213.

Kocak, O. and Ekiz, B. (2006): Effects of left displaced abomasum, ketosis and digestive disorders on milk yield in dairy cows. Bulg. J. Vet. Med., 9, 4: 273-280. 
Moore, G.R.; Riley, W.F. and Westcott, R.W. (1954): Displacement of the bovine abomasum. Veterinary Medicine 49: 49-51.

Naeini, A.T. and Rowshan, A. (2008): Surgery of the Abomasal Displacement: Right or Left Flank Approach? Iranian, J. Vet. 158-162.

Radostits, OM.; Gay, CC. and Hinchcliff, $K W$. (2007): Disease of abomasum. In: Veterinary Medicine, $10^{\text {th }}$ ed. Philadelphia, PA: W.B Saunders.353-374.

Radostits, O.M.; Gay, C.C.; Blood, D.C. and Hinchcliff, K.W. (2000): Veterinary medicine. $9^{\text {th }}$ ed. St. Louis: W.B. Saunders, 1877 p.

Radostits, O.M.; Gay, C.C.; Blood, D.C.; Hinchcliff, K.W. (2000): Veterinary medicine. $9^{\text {th }}$ ed. St. Louis: W.B. Saunders, $1877 \mathrm{p}$.

Ricken, M.; Hamann, H.; Scholz, H. and Distl, O. (2004): Genetische Analyse der Pra"valenz von Labmagenverlagerung und deren Beziehung $\mathrm{zu}$ Milchleistungsmerkmalen bei Deutschen Holstein Ku"hen. Deutsche Tiera"rztliche Wochenschrift 111, 366-370.

Saco, Y.; Fina, M. and Gime'nez, M. (2008): Evaluation of serum cortisol, metabolic parameters, acute phase proteins and faecal corticosterone as indicators of stress in cows. Vet. J. 177: 439-441.

Staric, J.; Biricik, H.S.; Aksoy, G. and Zadnik, T. (2010): Surgical Treatment of Displaced Abomasum in Cattle Using Ljubljana Method. Acta. Vet. Brno, 79: 469-473.

Steiner, A. (2006): Surgical treatment of the left displacement of the abomasum an update. In: XXIV. World BuiatricsCongress, Nice, France, pp. 165-169.

Plus, S. (1995): Guid to Statistical and Mathematical Analyses. Version 303, StatSci. Division, Mathsoft, Inc. Seattle Washington USA.

Van Winden, S.L.C. and Kuiper, R. (2003): Left displacement of the abomasum in dairy cattle: recent developments in epidemiological and etiological aspects. Veterinary Research 34: 47-56.

Wittek, T.; Constable, PD. and Morin, DE. (2005): Ultrasonographic assessment of change in abomasal position during the last three months of gestation first three months of lactation in HolsteinFriesian cows. J. Am. Vet. Med. Assoc. 227: 1469-1475. 
Zadnik, T. (2003): A comparative study of the hemato-biochemical parametrs between clinically healthy cows and cows with displacement of the abomasum, Acta Veterinaria, 53: 297-309.

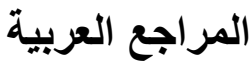

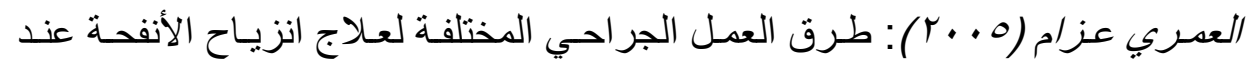

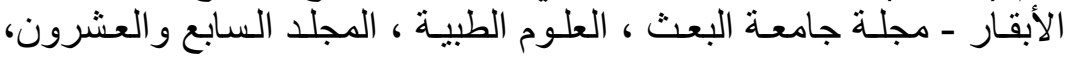

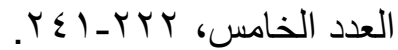

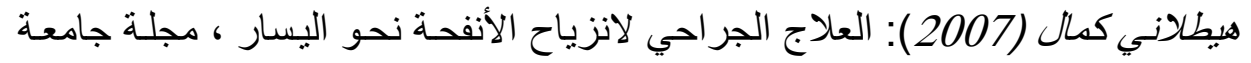

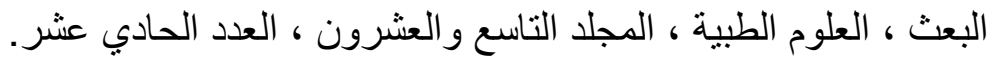

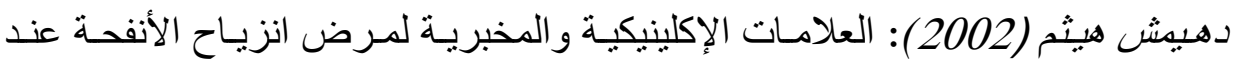
الأبقار ، رسالة ماجستير ، كلية الطب البيطري ، جامعة البعث. 